\title{
Delirium postcardiac surgery: Intellectual insufficiency and insufficiently understood
}

\author{
Ming-Sing Si, MD
}

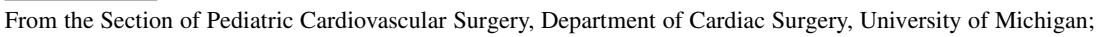
and C.S. Mott Children's Hospital, Ann Arbor, Mich.

Disclosures: Author has nothing to disclose with regard to commercial support.

Received for publication Sept 5, 2017; accepted for publication Sept 8, 2017; available ahead of print Oct 5, 2017. Address for reprints: Ming-Sing Si, MD, C.S. Mott Children's Hospital, SPC 4204, 1540 E. Hospital Drive, Ann Arbor, MI 48109-4204 (E-mail: mingsing@umich.edu).

J Thorac Cardiovasc Surg 2018;155:276-7

0022-5223/\$36.00

Copyright $(c) 2017$ by The American Association for Thoracic Surgery

https://doi.org/10.1016/j.jtcvs.2017.09.037

Over the past few decades and as a product of cost containment and patient satisfaction, fast-track cardiac surgery has become standard of care. The goal of fast-track cardiac care after cardiac surgery is for rapid recovery and decreased intensive care unit (ICU) and hospital length of stay. The major strategies to accomplish this goal include low-dose opioid use during general anesthesia and time-directed protocols for early extubation. ${ }^{1}$ Although these strategies are effective in decreasing ICU stay, other factors that impact the failure to fast track (FTF) after adult cardiac surgery are not as well understood.

In this issue of the Journal, Lee and colleagues ${ }^{2}$ present $^{-}$ their results on a study of the impact of delirium on FTF after cardiac surgery. These investigators evaluated the effects of the motor subtypes of delirium in FTF after cardiac surgery. Their objectives were to characterize the motor subtypes of delirium over different sedation levels and to determine whether there was any association with FTF. They hypothesized hypoactive delirium would be associated most strongly with FTF after cardiac surgery because of its worse prognosis for morbidity or mortality determined in previous studies of mixed ICU populations. ${ }^{3-5}$

To accomplish their objectives and evaluate their hypothesis, the investigators conducted a prospective study of 600 adult patients undergoing cardiac surgery. They used a standard definition of motor subtypes of delirium in their study: hyperactive (pure agitation), hypoactive (pure lethargy), and mixed (both agitation and lethargy). Determination of the motor subtypes of delirium were determined by trained bedside nurses who used a standard method involving the Confusion Assessment Method for the ICU and Richmond Agitation-Sedation Scale. ${ }^{3}$ FTF was defined as failure to extubate within 48 hours, readmission to the ICU, and mortality within 30 days.

These investigators found that delirium occurred in $13.8 \%$ of their patients, of which $4.3 \%, 4.0 \%$, and $5.5 \%$ were of the hypoactive, hyperactive, and mixed motor subtypes, respectively. They also determined that $14.7 \%$ of patients had FTF after cardiac surgery. There was a significant

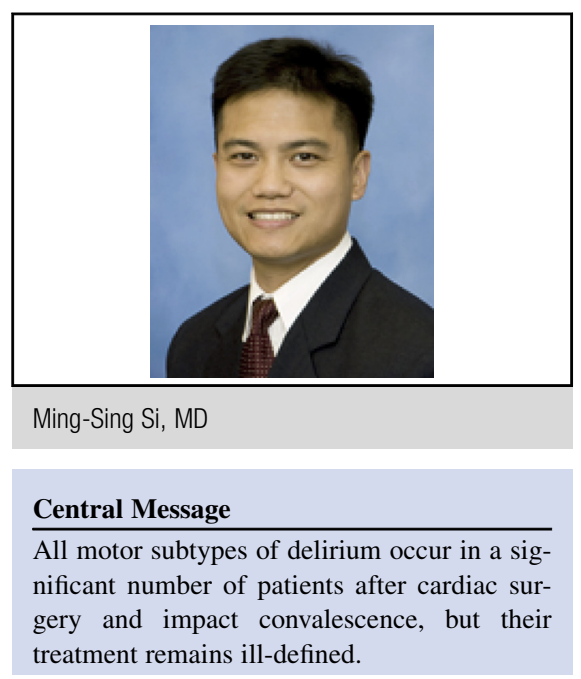

See Article page 268.

association between delirium (and all motor subtypes) and FTF. Hypoactive and mixed delirium subtypes were associated with a longer ICU stay. There were no identified associations between delirium motor subtype and ICU readmission or 30-day mortality. The authors conclude that delirium is an important complication after adult cardiac surgery that can impact postoperative recovery. They also conclude that all motor subtypes of delirium are important in contributing to FTF, and thus treatment strategies for delirium should be individualized to the specific subtype.

Their conclusion brings us to the discussion of the treatment of postoperative delirium and its subtypes. Delirium is an acute neuropsychiatric disturbance characterized by fluctuating alterations in cognition and consciousness. Dementia can be viewed as a chronic decline in mental ability and is a strong risk factor for developing delirium. ${ }^{6}$ It should be noted that the prevalence of dementia before cardiac surgery was not assessed by Lee and colleagues. ${ }^{2}$ Delirium and dementia can be viewed as "intellectual insufficiency," akin to other types of end-organ (eg, renal) insufficiency encountered in the acute (postcardiac surgery) or chronic settings. The pathogenesis of delirium has not been elucidated in detail but can be conceptualized as secondary to a direct brain insult (eg, hypoxia, sedative/anesthetic medications, and stroke) or as an aberrant stress response to an insult outside of the central nervous system (eg, sepsis). Furthermore, the differences in the pathogenesis of the different motor subtypes of delirium remain unknown. 
Not surprisingly, prevention and treatment strategies for delirium often are based on medical tradition and not on convincing evidence from clinical trials. Because of this lack of understanding and insight, delirium represents a major unmet medical need. ${ }^{7}$

Although Lee and colleagues ${ }^{2}$ don't elaborate on any new treatment strategies for delirium and its motor subtypes, their carefully conducted study does bring attention to this important condition that is not infrequent after cardiac surgery in both adults and children. ${ }^{8}$ Certainly, seemingly uncomplicated cardiac surgery can lead to direct brain insult (eg, potentially after hypothermic circulatory arrest $^{9}$ ) or an aberrant stress response to inflammatory cytokines ${ }^{10}$ that are beyond our means of routine detection. Continued research into the cellular and molecular mechanisms that incite delirium are needed so that more precise assessment (beyond Confusion Assessment Method for the ICU and Richmond Agitation-Sedation Scale methods) and treatment approaches can be developed. Furthermore, better understanding of the pathogenesis of delirium will allow potential improvements on how cardiac surgery and cardiopulmonary bypass are performed.

\section{References}

1. Wong WT, Lai VK, Chee YE, Lee A. Fast-track cardiac care for adult cardiac surgical patients. Cochrane Database Syst Rev. 2016;9:CD003587.

2. Lee A, Mu JL, Chiu CH, Gin T, Underwood MJ, Joynt GM. Effect of motor subtypes of delirium in the intensive care unit on fast-track failure after cardiac surgery. J Thorac Cardiovasc Surg. 2018;155:268-75.e1.

3. Robinson TN, Raeburn CD, Tran ZV, Brenner LA, Moss M. Motor subtypes of postoperative delirium in older adults. Arch Surg. 2011;146:295-300.

4. Stransky M, Schmidt C, Ganslmeier P, Grossmann E, Haneya A, Moritz S, et al. Hypoactive delirium after cardiac surgery as an independent risk factor for prolonged mechanical ventilation. J Cardiothorac Vasc Anesth. 2011;25:968-74.

5. Peritogiannis V, Bolosi M, Lixouriotis C, Rizos DV. Recent insights on prevalence and corelations of hypoactive delirium. Behav Neurol. 2015;2015:416792.

6. Ahmed S, Leurent B, Sampson EL. Risk factors for incident delirium among older people in acute hospital medical units: a systematic review and meta-analysis. Age Ageing. 2014;43:326-33.

7. Maclullich AM, Anand A, Davis DH, Jackson T, Barugh AJ, Hall RJ, et al. New horizons in the pathogenesis, assessment and management of delirium. Age Ageing. 2013;42:667-74.

8. Patel AK, Biagas KV, Clarke EC, Gerber LM, Mauer E, Silver G, et al. Delirium in children after cardiac bypass surgery. Pediatr Crit Care Med. 2017;18:165-71.

9. Fleck TM, Czerny M, Hutschala D, Koinig H, Wolner E, Grabenwoger M. The incidence of transient neurologic dysfunction after ascending aortic replacement with circulatory arrest. Ann Thorac Surg. 2003;76:1198-202.

10. Kazmierski J, Banys A, Latek J, Bourke J, Jaszewski R. Raised IL-2 and TNFalpha concentrations are associated with postoperative delirium in patients undergoing coronary-artery bypass graft surgery. Int Psychogeriatr. 2014;26: 845-55. 